\title{
REALIDAD VIRTUAL COMO TECNOLOGIA ASISTENCIAL A DEPORTISTAS CON MOVILIDAD REDUCIDA EN SILLA DE RUEDAS
}

\author{
Iván Cáceres Rodríguez, Emiliano Pérez, Inés Tejado, Blas M. Vinagre, Pilar Merchán, Santiago Salamanca \\ Universidad de Extremadura, Escuela de Ingenierías Industriales, Avenida de Elvas, s/n, 06006 Badajoz \\ Email: ivcaceres@alumnos.unex.es, \{emilianoph,itejbal,bvinagre,pmerchan,ssalamanca $\} @$ unex.es
}

\begin{abstract}
Resumen
Son numerosas las tecnologías que se han ido incorporando al ámbito deportivo paralímpico en los últimos años con el objeto de desarrollar sistemas de asistencia que permitan mejorar el rendimiento de los deportistas, como por ejemplo, aquellos con movilidad reducida en silla de ruedas. Entre ellas, cabe destacar el potencial de la realidad virtual para crear mundos interactivos como medio de inmersión. Este trabajo describe el proceso de integración de unos guantes de datos en el simulador de movimiento de la Universidad de Extremadura para la creación de mundos virtuales para la asistencia a este tipo de deportistas con movilidad reducida con el objeto de mejorar, en un futuro, su rendimiento deportivo. Para ilustrar el potencial de este simulador, se presenta una aplicación de baloncesto desarrollada a modo de ejemplo.
\end{abstract}

Palabras Clave: ingeniería, simulador, realidad virtual, asistencia, deportista, silla de ruedas, guantes.

\section{INTRODUCCIÓN}

Son muchos los desafíos a los que se enfrentan las personas con movilidad reducida en silla de ruedas en su día a día, entre los que se pueden mencionar la falta de familiaridad con la navegación con la silla en lugares públicos, el miedo a lo desconocido y la falta de confianza. Estos y otros desafíos también aparecen en el ámbito del deporte paralímpico.

Desde hace unos años, los avances en realidad virtual (VR de su terminología inglesa) han abierto nuevas posibilidades en el deporte, y en especial en el paralímpico. En este sentido, la ingeniería de las tecnologías asistenciales y de la rehabilitación se consideran indispensables para que los sistemas de ayuda a la movilidad se adapten a las necesidades del usuario. Con esta premisa, el diseño y la realización de pruebas que simulen situaciones reales que proporcionen datos para la adaptación al usuario de esas asistencias técnicas son primordiales para su éxito. Es por ello que en los últimos años ha aumentado el interés en el desarrollo de métodos de evaluación de las capacidades motoras y cognitivas que faciliten el proceso de elección de los sistemas de ayuda técnica basados en VR adecuados. Algunos ejemplos de prototipos de simuladores de sillas de ruedas para el caso particular de personas con movilidad reducida pueden encontrarse en [4,6-12, $18]$, o revisiones en $[1,16]$. Sin embargo, entre ellas, las aplicaciones deportivas son limitidas.

Este trabajo completa el proceso de integración de los equipos que constituyen el simulador de movimiento de la Universidad de Extremadura para la creación de mundos virtuales asistenciales para deportistas con movilidad reducida, especialmente centrados en ejercicios con las manos. Conviene resaltar que este trabajo es la continuación de otro previo en el que se puso en marcha del simulador y se crearon mundos virtuales para la interacción con sillas de ruedas [17]. Partiendo del mismo, en este artículo se describe el proceso de integración de guantes de datos para capturar la flexión de los dedos de las manos que permita desarrollar aplicaciones virtuales en las que la captura y evaluación de los movimientos con las manos sean de importancia. A modo de ejemplo, se presenta una pista de baloncesto virtual. Para ello, se utilizará el sistema de captura óptico del simulador para capturar el cuerpo completo del usuario, incluidas las manos.

El objetivo final de este trabajo es, en un futuro, el diseño y la aplicación de tecnologías asistenciales que permitan mejorar la calidad de vida de este tipo de personas con movilidad reducida. Asimismo se pretende que el simulador pueda servir de ayuda al diseño de infraestructuras de edificios que permitan una mejor adaptación para los usuarios en silla de ruedas.

El resto del artículo se encuentra estructurado como se describe a continuación. El apartado 2 describe brevemente los principales equipos del simulador. El apartado 3 resume los procesos de integración de la estación de trabajo con el sistema de captura óptico, la plataforma de movimiento y el sistema háptico, el casco de realidad virtual, y los guantes de datos 
mediante Unity para la creación de los mundos virtuales. El apartado 4 presenta una de las aplicaciones desarrolladas. Las conclusiones de este trabajo, así como líneas de trabajo futuro, se discuten en el apartado 5.

\section{EL SIMULADOR}

El simulador está formado por los siguientes equipos:

- una estación de trabajo (PC);

- una interfaz con la silla de ruedas, formada por una plataforma de movimiento, otra háptica para la silla y elementos de acceso;

- sistemas de captura corporal óptico y guante de datos;

- dispositivos de visualización, en concreto, una pantalla 3D y un casco de realidad virtual.

La Figura 1 muestra las relaciones entre los equipos del simulador. En los siguientes subapartados se detallarán las características de cada uno de ellos. La descripción completa puede encontrarse en [17].

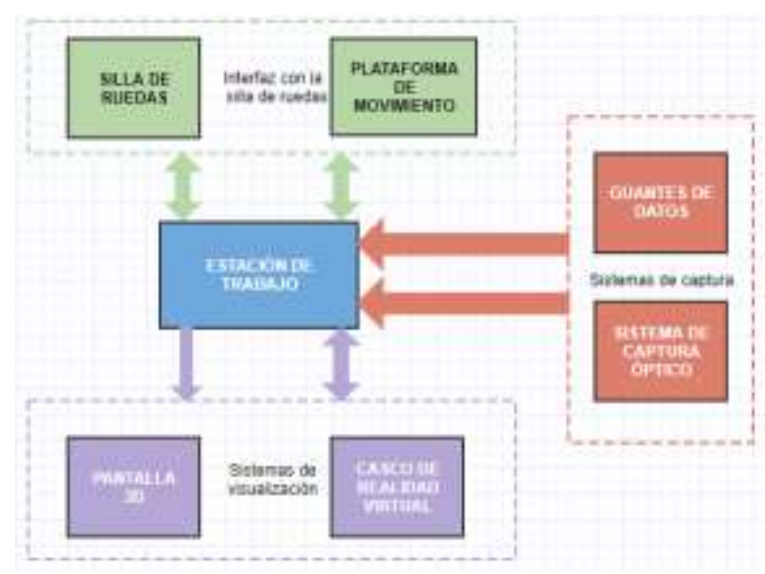

Figura 1: Simulador y equipos que lo conforman

\subsection{ESTACIÓN DE TRABAJO}

La estación de trabajo es un PC que hace las funciones de "cerebro" de todo el simulador, ya que se encarga de recoger la información proporcionada por todos y cada uno de los elementos y procesarla de forma adecuada, así como de permitir la comunicación entre estos. Se ha optado por utilizar un ordenador y el motor de videojuegos Unity como eje principal, dado lo mucho que facilita la incorporación de los distintos modelos físicos de nuestro mundo y poder adaptarlos a un entorno virtual creado en la propia plataforma.

Para que el software trabaje adecuadamente con los elementos del simulador lo único que se necesita son las correspondientes librerías, dado que los scripts necesarios para su funcionamiento se crean en Unity usando el lenguaje de programación C\#.

Como es evidente, es necesario un equipo con una serie de características mínimas para poder coordinar todo y a la vez mostrar los elementos con la calidad gráfica mínima necesaria. Cabe recalcar que uno de los objetivos de este trabajo es hacer que el usuario tenga una experiencia $100 \%$ inmersiva, para lo cual es necesario que todo se vea bien y con una calidad alta, para ello es recomendable utilizar tarjetas gráficas con funcionalidad VR ya incorporadas. En el simulador se utilizan simultáneamente dos tarjetas gráficas: Nvidia GTX970 y Nvidia Quadro K5200.

\subsection{INTERFAZ CON LA SILLA DE RUEDAS}

Una de las partes más importantes y novedosas del simulador es la interfaz con la silla de ruedas. Como se ha comentado, la integran una plataforma de movimiento de seis grados de libertad y un sistema háptico de rodillos accionados por motores sobre los que descansan las ruedas de la silla, a fin de registrar el movimiento realizado por estas y/o ejercer una aceleración o freno sobre el movimiento de las mismas. La Figura 2(a) muestra una foto de la interfaz.

La silla de ruedas utilizada en el simulador es el modelo deportivo All Court de Quickie. No obstante, la plataforma cuenta también con un elevador automático para que los usuarios puedan acceder ella con su propia silla de ruedas. Éste puede verse en la Figura 2(b).

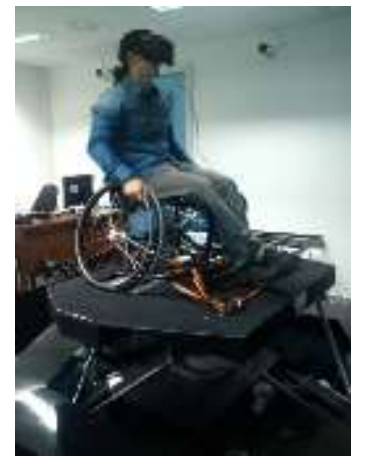

(a)

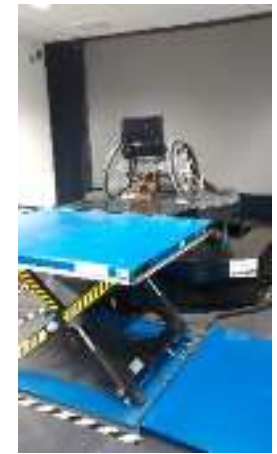

(b)
Figura 2: Interfaz con la silla de ruedas: (a) Plataforma de movimiento, sistema háptico y silla (b) Elementos de acceso a la plataforma

\subsection{SISTEMAS DE CAPTURA}

Para registrar los movimientos realizados por el usuario, el simulador dispone de un sistema óptico de 
captura del movimiento corporal (en este caso del tronco superior, salvo la flexión de los dedos de la mano) de la marca Optitrack [14], y de unos guantes de datos 5DT [5] para el registro del movimiento de los dedos (véase la Figura 3 ).

Los datos capturados por el sistema de Optitrack serán transmitidos al avatar del mundo virtual en Unity en tiempo real gracias a su software Motive [15], que también permite calibrar y configurar el espacio de trabajo. Por su parte, los datos de los guantes son transmitidos a Unity mediante los scripts que proporciona el fabricante, tal y como se describe en detalle en el subapartado 3.4.

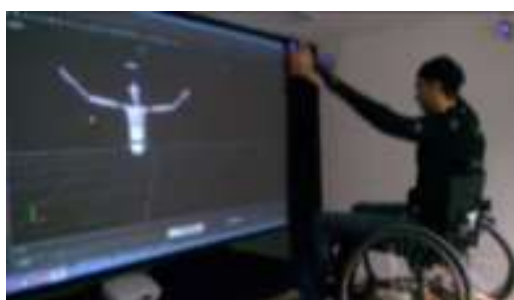

(a)

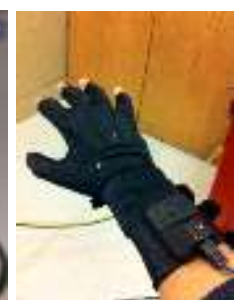

(b)
Figura 3: Sistemas de captura: (a) Sistema óptico de captura corporal (b) Guante de datos

\subsection{SISTEMAS DE VISUALIZACIÓN}

A la hora de visualizar el mundo virtual, el simulador dispone del casco de realidad virtual Development Kit 2 (DK2) de Oculus Rift [13] de la Figura 4 para el usuario, y de una pantalla $3 \mathrm{D}$ de grandes dimensiones para que tanto el equipo técnico como cualquier persona presente en la sala también pueda observar la aplicación en funcionamiento y dar las indicaciones oportunas al usuario.

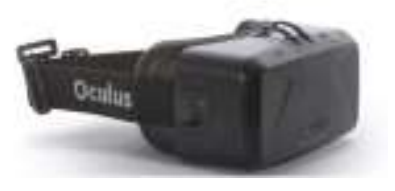

Figura 4: Casco de realidad virtual Development Kit 2 (DK2) de Oculus Rift

\section{INTEGRACIÓN DE EQUIPOS EN EL MUNDO VIRTUAL}

En este apartado se describen todos los procesos de adaptación llevados a cabo para integrar los distintos equipos para la creación de los mundos virtuales mediante Unity, haciendo especial hincapié en los guantes de datos. Consúltese [17] para más detalles acerca de la integración del sistema de captura óptico, la plataforma de movimiento/sistema háptico y el casco de realidad virtual.

\subsection{SISTEMA DE CAPTURA ÓPTICO}

El proceso de integración del sistema de captura corporal óptico OptiTrack con Unity se ha realizado mediante el plugin que proporciona el fabricante siguiendo los siguientes pasos:

1. Definición en Motive de un esqueleto, utilizando uno de los preconfigurados (full body de 27 marcadores).

2. Adaptación de sistemas de referencia.

3. Activación de la transmisión de datos en streaming desde Motive mediante protocolo Multicast.

4. Carga del plugin en Unity y asignación del script a un esqueleto de Unity que ha de tener una estructura jerárquica determinada, como la mostrada en la Figura 5.

5. Enlace entre el esqueleto Unity y el esqueleto Motive (asignando el mismo nombre).

\subsection{PLATAFORMA DE MOVIMIENTO Y SISTEMA HÁPTICO}

En este caso, el procedimiento de integración de la plataforma y la silla de ruedas en Unity se ha llevado a cabo del siguiente modo:

- Para el modelado de la plataforma, se ha desarrollado un script a partir del motor de físicas PhysX de Unity que permite la comunicación bidireccional entre este software y la misma con dos funciones principales: 1) detectar los movimientos que realiza la silla en Unity, así como las aceleraciones que experimente el modelo virtual y transmitirlos a la plataforma; y 2) capturar las órdenes de movimiento que da el usuario, es decir, el ángulo y la velocidad de giro de cada una de las ruedas, a través del sistema de rodillos de la plataforma. Asimismo, este script permite la configuración de una serie de parámetros, tanto de la plataforma como del mundo virtual, entre los que conviene destacar la velocidad de los motores de la plataforma y los factores de corrección de la velocidad de los rodillos. Estos últimos permiten configurar la interacción entre el mundo virtual y el sistema de rodillos de tal modo que es posible caracterizar diferentes tipos de superficies en el mundo virtual mediante resistencia al movimiento o rozamiento con la superficie.

- Para la representación de la silla de ruedas, el modelo 3D se ha dividido en un chasis y las dos ruedas principales motrices, a los que se le ha asociado la propiedad denominada collider, que hace que el objeto sea tenido en cuenta dentro del motor de físicas para el cálculo de colisiones entre objetos. 

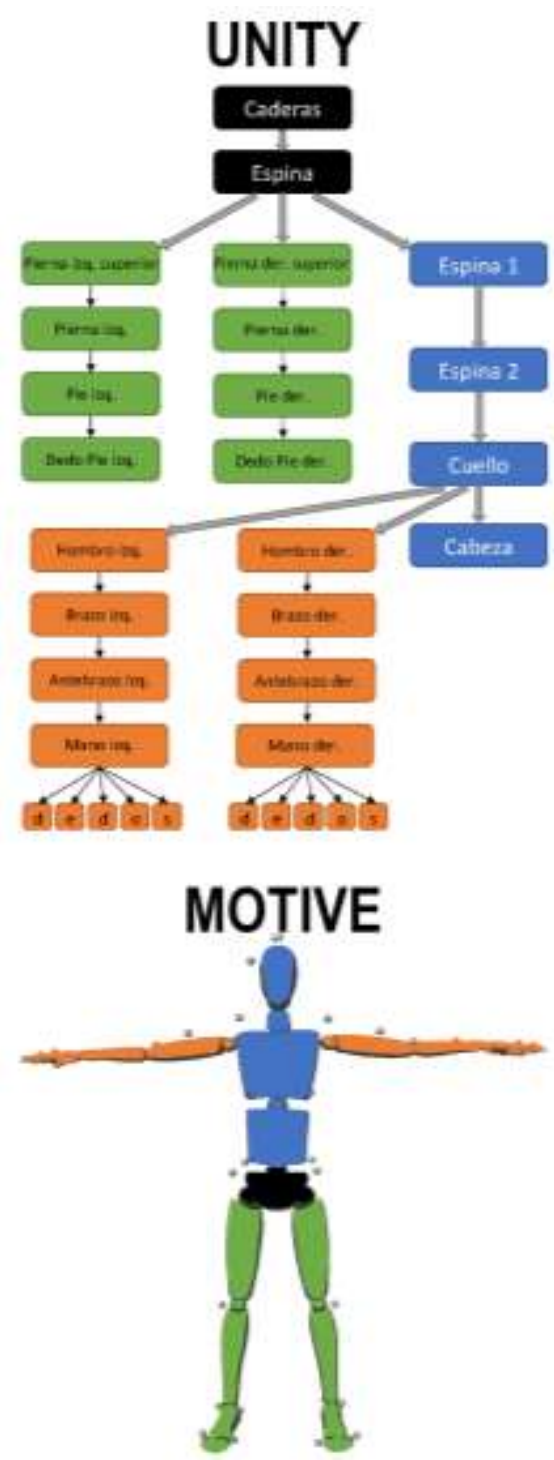

Figura 5: Correspondencia, representada con colores, entre la jerarquía del esqueleto del avatar de Unity y el esqueleto de Motive

\subsection{CASCO DE REALIDAD VIRTUAL}

La integración del casco de realidad virtual se ha llevado a cabo mediante la versión 1.13 del plugin proporcionado por Oculus Rift. Es importante resaltar que, a la hora de realizar el posicionamiento absoluto del mismo en el entorno virtual, se ha preferido utilizar el sistema de captura óptico, como ya se ha comentado, mediante un conjunto de 6 marcadores asimétricos.

\subsection{GUANTES DE DATOS}

Este apartado está dedicado a la descripción de los guantes de datos. Asimismo se explica cómo se ha realizado el proceso de integración de los guantes en Unity y el posicionamiento absoluto.

\subsubsection{Descripción}

Otros de los elementos que sirven como interfaz entre el usuario y el simulador son los guantes de datos, que permiten detectar y registrar la flexión de los dedos del usuario, para un posterior análisis y diagnóstico si así fuera el caso de aplicación tratado. En concreto, el modelo de guantes es de la marca 5DT, que cuenta con 5 sensores de flexión por cada mano (uno por dedo). Como se puede ver en la Figura 6, estos guantes disponen de un kit inalámbrico Bluetooth para el envío de los registros, evitando así la necesidad de usar cables con la estación de trabajo, aunque añade la necesidad de incorporar unas baterías al traje que viste el usuario.

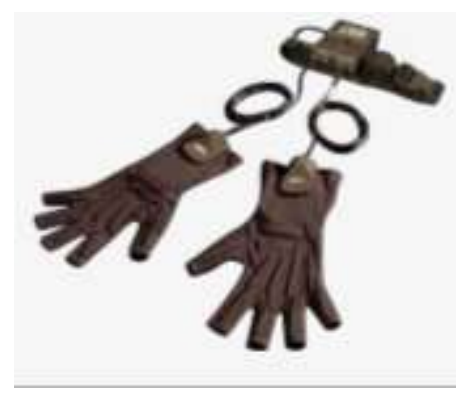

Figura 6: Guantes de datos con 5 sensores de flexión

Cabe señalar que estos guantes no permiten la detección de la posición de las manos del usuario (posicionamiento absoluto), cuestión que es esencial para poder desarrollar una aplicación en la que el usuario pueda interactuar con el mundo virtual. Para solucionarlo, el uso del guante se combina con el sistema de captura corporal de Optitrack.

\subsubsection{Integración en Unity}

El fabricante proporciona unas librerías en $\mathrm{C}++$ para programar el uso del guante. Dado que Unity permite el uso de scripts en C\# o Javascript, hubo que reescribir todas las librerías y adaptarlas de $\mathrm{C}++$ a C\#. Los scripts obtenidos básicamente realizan una búsqueda de los guantes conectados a los puertos USB y puertos COM de la estación de trabajo. Una vez detectados, Unity recibe la información de flexión de cada uno de los dedos en diferentes señales escaladas entre 0 y 1 , correspondiendo el 0 a un dedo estirado (mano en reposo o relajada) y el 1 a una flexión completa (puño cerrado).

Para conseguir la representación en 3D de la flexión de los dedos en la mano virtual en tiempo real se han llevado a cabo como se describe a continuación:

1. Diseño del modelo o malla 3D de una mano.

2. Asociación de un esqueleto con las jerarquías entre las articulaciones que la componen de 
acuerdo con la estructura mostrada en la Figura 7(a) (proceso denominado rigging [20]).

3. Traducción de las señales de los guantes a rotaciones de las articulaciones de los dedos virtuales. De esta forma, las señales procedentes de los guantes se asocian a la rotación de las articulaciones del esqueleto.

El movimiento de estas articulaciones deformará la malla $3 \mathrm{D}$ de la mano que adoptará la posición que tenga su esqueleto.

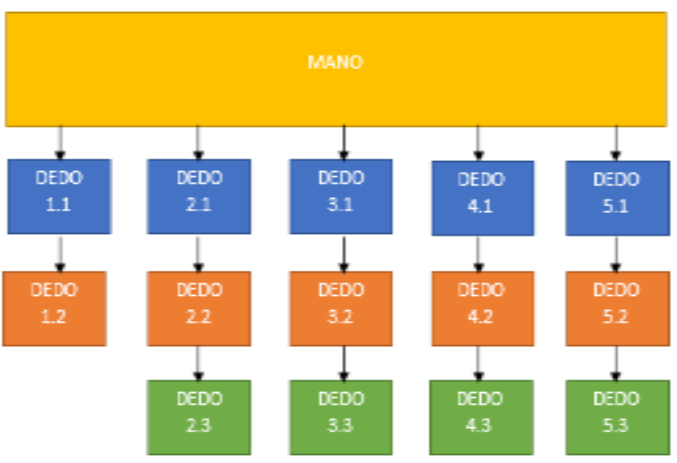

(a)

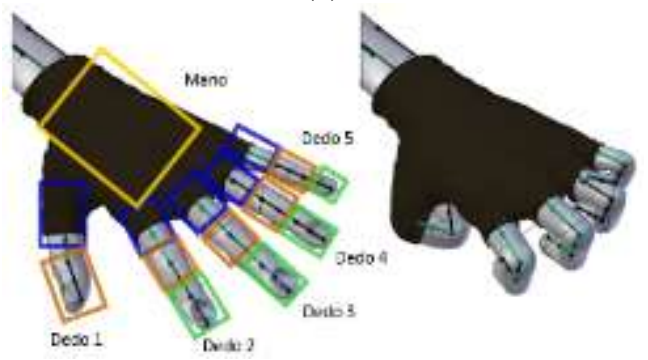

(b)

Figura 7: Esquemas de integración de los guantes de datos en Unity: (a) Estructura jerarquizada de la malla de la mano (b) Representación de las dos posiciones extremas para la animación de la mano con los dedos flexionados y los dedos extendidos

Para asociar las señales recibidas a posiciones específicas del esqueleto, Unity cuenta con las herramientas Animator [2] y Animation [3]. Estas herramientas permiten animar el movimiento o rotación de elementos 3D. Específicamente se utiliza el método conocido como Blending Tree, que permite alternar de forma fluida entre dos o más animaciones. Un ejemplo muy típico de uso consiste en hacer que un personaje pase de andar a correr en función de una variable, la velocidad. Este método también permite crear animaciones estáticas, es decir, posiciones fijas y hacer que se cambie entre ellas en función de una variable preestablecida. Por lo tanto, para animar la flexión de los dedos se han seguido los siguientes pasos:

1. Creación, individualmente para cada uno de los dedos, de todas las animaciones de dedo extendido y flexionado.
2. Creación de un Blending Tree "padre" de cada mano del que cuelgan receptores correspondientes a cada dedo. Después, de cada dedo parten dos posibles caminos asociados a las animaciones del paso anterior: dedo totalmente extendido o dedo totalmente flexionado (Figuras 7(b) y 8).

3. Asociación del script que recoge los datos del guante a la malla jerarquizada (con rig) de la mano.

4. Asociación de las señales recibidas del guante al Blending Tree creado en el segundo paso. Además, a cada dedo se le ha añadido un valor de ganancia personalizable, para ajustar manualmente la señal escalada recibida (entre 0 y 1) a la animación de la flexión del dedo.

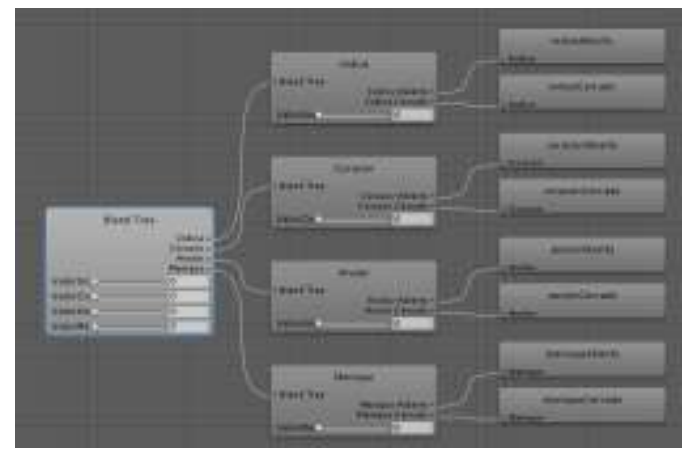

Figura 8: Ejemplo de Blending Tree

Unity hace que la transición entre las animaciones, dedo extendido y dedo flexionado, sea lineal. Es posible que alguna malla o modelo $3 \mathrm{D}$ de la mano, debido a su forma, de lugar a que, en algunas posiciones, ésta tenga auto-intersecciones. Para ello, se debe afinar aún más la transición incluyendo pasos intermedios (posiciones de la malla ajustadas para evitar auto-intersecciones), para así forzar el paso por esa posición durante el proceso de transición entre la flexión y la extensión del dedo.

Una vez conseguida la conexión entre las flexiones de los guantes con la animación de las mallas 3D de las manos, se tiene que añadir a la mano las capacidades de interacción con elementos del mundo virtual. El objetivo, en el caso particular de esta aplicación, es que el usuario pueda interaccionar con una pelota virtual: agarrarla, botarla, lanzarla, etc. Esto puede llevarse a cabo mediante el uso de las capacidades del motor de físicas de Unity una vez definidos los colliders asociados a la malla de la mano, que permitirán detectar cuándo otro elemento 3D colisiona o interseca con esa malla (cuando sobrepase los límites del correspondiente collider).

En concreto, el método que se ha empleado, por consumir menor cantidad recursos, consiste en definir colliders geométricamente sencillos para 
cubrir el objeto. El más simple que nos proporciona Unity es una caja 3D. Así, se ajustaron manualmente un collider caja para cada una de las falanges, otro para la palma de la mano, otro para el antebrazo del avatar y un último para el torso, de manera que todas las zonas importantes estuvieran cubiertas, tal y como se ilustra en la Figura 9. Por último, para que el motor de físicas compute las colisiones con estos colliders es necesario añadir el componente "Rigidbody" a la malla 3D.

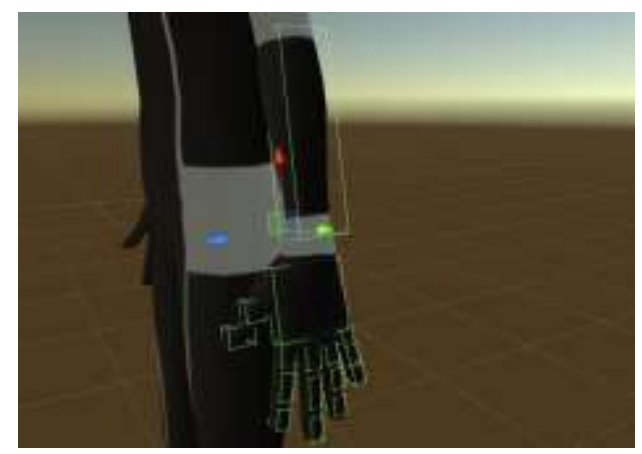

Figura 9: Colliders de la mano y antebrazo

\subsubsection{Posicionamiento absoluto}

Como se ha comentado, el guante 3D de 5DT no incluye ningún tipo de tecnología para detectar la posición de las manos del usuario. Para suplir esa carencia se hace uso del sistema de captura óptico. En concreto se ha seguido los pasos que se enumeran a continuación para lograr el posicionamiento absoluto de las manos en el simulador:

1. Se añaden marcadores ópticos a cada guante, distribuidos de forma asimétrica en las dos manos, para que se diferencie claramente la mano derecha de la izquierda.

2. En Motive, se asocia un sólido rígido a cada grupo de marcadores correspondiente a cada mano y se asocia un identificador ID a cada uno de esos sólidos.

3. Se activa la transmisión de datos desde Motive mediante streaming.

4. Se carga el plugin de Optitrack en Unity y se asigna el script del sólido rígido a cada una de las manos, respetando el indicador "Rigid Body Id" para que coincida con el de Motive.

\subsection{INTEGRACIÓN DE TODOS LOS ELEMENTOS}

Como se ha comentado en la introducción, el objetivo del presente trabajo es integrar el uso de los guantes en el simulador y aplicar todo el sistema a deportistas en silla de ruedas. Por tanto, para la aplicación final se utilizará el sistema Optitrack para capturar el cuerpo completo del usuario, incluidas las manos, siguiendo los siguientes pasos:

1. Definición en Motive de un esqueleto, utilizando uno de los preconfigurados (full body de 32 marcadores).

2. Definición en Motive de un sólido rígido que se asociará a los marcadores del casco de realidad virtual.

3. Activación de la transmisión de datos en streaming desde Motive mediante protocolo Multicast.

4. Carga del plugin en Unity y asignación del script a un esqueleto de Unity que ha de tener una estructura jerárquica determinada, como la mostrada en la Figura 5.

5. Creación de estructura de colliders en dedos, manos, antebrazo y torso.

6. Enlace entre el esqueleto Unity y el esqueleto Motive (se les debe asignar el mismo nombre), así como entre el casco de VR en Unity y el sólido rígido correspondiente de Motive.

7. Inclusión de los scripts y Blending Trees a las manos del esqueleto de Unity.

A modo de resumen, en la Figura 10 se representa un esquema con las relaciones entre los diferentes programas empleados.

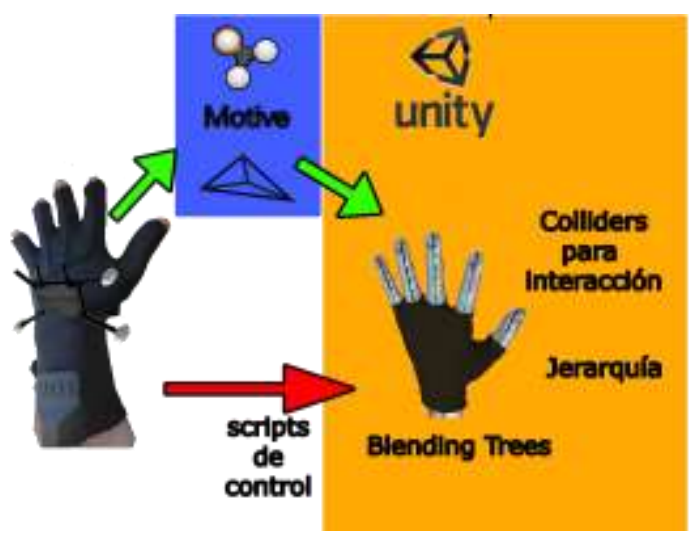

Figura 10: Esquema que resume las relaciones entre el guante de datos con los diferentes programas implicados la simulación

\section{EJEMPLO DE APLICACIÓN DESARROLLADA}

Este apartado explica la aplicación que se ha desarrollado para el simulador, con objeto de mostrar las posibilidades de uso relacionadas con el análisis de juego y diagnóstico de deportistas con movilidad reducida en silla de ruedas.

Se trata de una aplicación básica que sirve como demostración de las posibilidades de este tipo de tecnología y hace uso de todos los dispositivos 
funcionando simultáneamente: plataforma de movimiento, sistema de captura óptico, guantes de datos y casco de VR.

El usuario accede a la plataforma de movimiento con su silla de ruedas y vistiendo el traje, los guantes y el casco VR. Los técnicos pueden observar lo que está haciendo el usuario mediante la pantalla 3D.

Dado que el contexto de la aplicación es el trabajo con deportistas discapacitados se ha decidido aplicarlo a jugadores de baloncesto en silla de ruedas. El escenario virtual que se ha diseñado consiste en una pista de baloncesto a escala real, mostrada en la Figura 11.

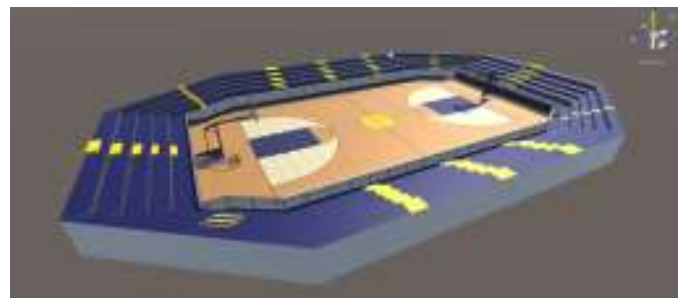

Figura 11: Pista virtual de baloncesto

Las interacciones del usuario serán con la pelota, también creada en el entorno virtual y con efectos de sonido añadidos para conseguir una mayor sensación de inmersión.

La aplicación permite el libre desplazamiento del usuario por toda su superficie limitado nada más por unas paredes invisibles para que ni él ni la pelota se salgan de la pista en ningún momento. Además, a modo de minijuego se ha añadido un marcador que se encargará de llevar la cuenta de todas las canastas encestadas por el usuario. Para poder realizar este conteo se ha creado un collider invisible bajo la canasta, además de un script correspondiente para que cada vez que la pelota lo cruce añada un punto. Por el momento, se ha decido no entrar en profundidad en las reglas del baloncesto y distinguir, por ejemplo, entre dobles o triples, contando todas por igual, ya que el objeto de esta aplicación no es tanto ser un simulador de baloncesto válido sino mostrar un posible uso. En la Figura 12 se aprecia el collider visible bajo la canasta.

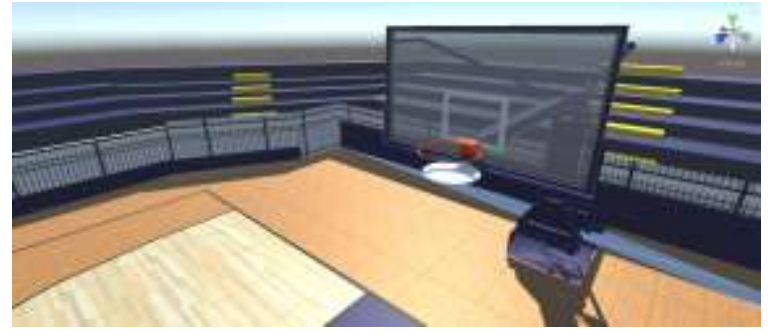

Figura 12: Collider visible del marcador

\section{CONCLUSIONES}

Este trabajo ha descrito el proceso de integración de unos guantes de datos en el simulador de movimiento de la Universidad de Extremadura para la creación de mundos virtuales para la asistencia a este tipo de deportistas con movilidad reducida con el objeto de mejorar, en un futuro, su rendimiento deportivo. Se ha presentado una aplicación de baloncesto desarrollada a modo de ejemplo.

Como trabajos futuros se plantean las siguientes líneas: 1) desarrollo de aplicaciones específicas para mejorar los rendimientos de los deportistas o incluso como entretenimiento en la industria del ocio; y 2) recogida de los datos proporcionados por los guantes a fin de tener información sobre los movimientos de la mano realizados por los pacientes y aquellos en los que tengan mayores dificultades para mejorar en lo posible su calidad de vida.

\section{Agradecimientos}

Este trabajo ha sido parcialmente financiado por Fondos FEDER (Programa Operativo FEDER de Extremadura 2014-2020), a través de la Ayuda a Grupos de la Junta de Extremadura.

Parte del equipamiento utilizado en este trabajo fue adquirido gracias al Programa Operativo FEDER de I+D+i por y para el Beneficio de las EmpresasFondo Tecnológico 2007-2013 del Ministerio de Economía y Competitividad a través del proyecto "Centro de Tecnificación del Deporte Paralímpico" (DEPATech).

\section{English summary}

\section{VIRTUAL REALITY AS ASSISTIVE TECHNOLOGY TO ATHLETES WITH LOCOMOTION DISABILITY IN A WHEELCHAIR}

\footnotetext{
Abstract

There are many technologies that have been incorporated into paralympic sports in recent years with the aim of developing assistance systems that improve the performance of athletes, such as those with locomotion disability in wheelchairs. Among them, it is worth noting the potential of virtual reality to create interactive worlds as a means of immersion. This work describes the process of integrating data gloves in the simulator of motion of the University of Extremadura for the creation of virtual worlds to assist such a kind of athletes with locomotion
} 
disability in order to improve, in the future, their sporting performance. To illustrate the potential of this simulator, a basketball application is developed as an example.

Keywords: engineering, simulator, virtual reality, assistive, athletes, wheelchair, gloves.

\section{Referencias}

[1] Abellard, P., Randria, I., Abellard, A., Ben Khelifa, M. M., Ramanantsizehena, P., (2010) "Electric Wheelchair Navigation Simulators: why, when, how?", Mechatronic Systems Applications, A. M. Donato Di Paola and G. Cicirelli (Ed.), InTech.

[2] Animator de Unity. Online: https://docs.unity3d.com/ScriptReference/An imator.html

Consultado el día: 14/03/2018.

[3] Animation de Unity. Online: https://docs.unity3d.com/ScriptReference/An imation.html

Consultado el día: 14/03/2018.

[4] Crichlow, L. R., Fernie, G. R., Campos, J. L., Grant, P. R., (2011) “A Full Motion Manual Wheelchair Simulator for Rehabilitation Research", Proc. 2011 RESNA/ICTA Conference on Advancing Rehabilitation Technologies for an Aging Society.

[5] Data gloves 5DT. Online: http://www.5dt.com/data-gloves/

Consultado el día: 24/01/2018.

[6] Fernández-Panadero, C., Cruz Barquero, V., Morán Núñez, D., Delgado Kloos, C., (2014) "PhyMEL-WS: Physically Experiencing the Virtual World. Insights into Mixed Reality and Flow State on Board a Wheelchair Simulator", Journal of Universal Computer Science, 20(12):1629-1648.

[7] Fernández-Panadero, C., Cruz Barquero, V., Morán Núñez, D., Delgado Kloos, C., (2013) "PhyMEL-WS Weelchair Simulator: A Preliminary Study to Increase Awareness about the Problems of Living the City in a Wheelchair", Proc. 3rd European Immersive Education Summit, pp. 210-221, UK.

[8] Gallien, P., Nicolas, B., Devigne, L., Pasteau, F., Lepape, L., Babel, M., Colin, A., Achille, S., Fraudet, B., (2017) "Virtual reality simulator for speeding up research on assisted power wheelchair navigation", 60:e93.

[9] Harrison, C. S., Grant, P. M., Conway, B. A., (2010) "Enhancement of a virtual reality wheelchair simulator to include qualitative and quantitative performance metrics", Assistive Technology, 22(1): 20-31.
[10] John, N. W., Pop, S. R., Day, T. W., Ritsos, P. D., Headleand, C. J., (2017) "The Implementation and Validation of a Virtual Environment for Training Powered Wheelchair Manoeuvres", IEEE Transactions on Visualization and Computer Graphics, In Press.

[11] Mahajan, H. P., Dicianno, B. E., Cooper, R. A., Ding, D., (2013) "Assessment of wheelchair driving performance in a virtual reality-based simulator", The Journal of Spinal Cord Medicine, 36(4):322-332.

[12] Niniss, H., Inoue, T., (2006) "Electric Wheelchair Simulator for Rehabilitation of Persons with Motor Disability", Proc. National Rehabilitation Centre for Persons with Disabilities Conference, Japón

[13] Oculus Rift. Online: https://www.oculus.com/rift/ Consultado el día: 04/02/2018.

[14] OptiTrack. Online: http://optitrack.com Consultado el día: 04/04/2018.

[15] OptiTrack. Motive documentation. Online: http://wiki.optitrack.com/index.php?title=Mo tive Documentation

Consultado el día: 04/04/2018.

[16] Pithon, T., Weiss, T., Richir, S., Klinger, E., (2009) "Wheelchair simulators: A review", Technology and Disability, 21(1):1-10.

[17] Sánchez Sánchez, C., Cidoncha Jiménez, M., Pérez, E., Tejado, I., Vinagre, B. M., (2017) "Simulador para la creación de mundos virtuales para la asistencia a personas con movilidad reducida en silla de ruedas", Actas de las XXXVIII Jornadas de Automática, pp. 1023-1030.

[18] Sergeeva, Y., (2017) A virtual reality based powered wheelchair simulator, Trabajo Fin de Máster, Western Michigan University.

[19] Tutorial sobre los Blending Tree de Unity: https://unity3d.com/es/learn/tutorials/topics/a nimation/blend-trees

Consultado el día: 10/05/2018.

[20] Miller, C., Arikan, O., Fussell, D., (2010) "Frankenrigs: Building Characteri Rigs from Multiple Sources", Proc. 2010 ACM SIGGRAPH Symposium on Interactive $3 D$ Graphics and Games, USA.

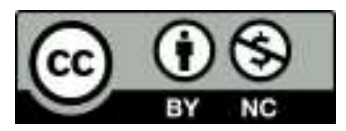

(C) 2018 by the authors. Submitted for possible open access publication under the terms and conditions of the Creative Commons Attribution CC-BY-NC 3.0 license (https://creativecommons.org/licenses/by-nc/3.0). 